\title{
Caracterización Mecánica del Fijador Multiaxial "ATLAS" para Fracturas de Huesos
}

\author{
Arlex Leyton* \\ José Jaime García** \\ Andrés Machado** \\ Andrés A. Echeverry****
}

* Ingeniero Mecánico - Estudiante de la Maestría en Ingeniería Mecánica - Universidad del Valle - Santiago de Cali, Colombia. E-mail:arleyton@hotmail.com

** Ph.D. - Ingeniero Mecánico - Profesor Titular Escuela de Ingeniería Civil y Geomática - Universidad del Valle Santiago de Cali, Colombia.

E-mail: josejgar@univalle.edu.co

*** Médico Ortopedista - Instituto de Seguros Sociales Santiago de Cali, Colombia

*** Médico Ortopedista - Profesor Titular Escuela de Medicina Universidad del Valle - Santiago de Cali, Colombia

E-mail: andresecheverry@calipso.com.co

Fecha de recepción: Junio 13 de 2003

Fecha de aprobación: Octubre 17 de 2003

\section{RESUMEN}

Se presenta la caracterización mecánica de un nuevo fijador unilateral multiaxial de bajo costo (ATLAS) desarrollado por nuestro grupo. Éste es un fijador de urgencias de doble barra, en el que se utilizan conectores que permiten seis grados de libertad entre las barras y los clavos que conectan, con lo cual se pueden activar también los seis grados de libertad entre los fragmentos de hueso. La caracterización mecánica se hizo con base en un procedimiento computacional y experimental para determinar la denominada matriz de flexibilidad interfragmentaria. El estudio también se realizó en otros dos fijadores importados y uno nacional ampliamente utilizado 
en el suroccidente de Colombia. La rigidez del nuevo fijador estuvo dentro del rango obtenido para los otros evaluados. Aún con una configuración de doble barra que le confiere estabilidad y rigidez al conjunto, el fijador ATLAS permite activar los seis grados de libertad entre los fragmentos de hueso, por lo cual el médico ortopedista puede reducir la fractura correctamente. Desde el punto de vista biomecánico, el fijador ATLAS se encuentra listo para ser evaluado clínicamente.

Palabras Clave: Fijadores externos, fracturas de huesos, desplazamientos interfragmentarios.

\section{ABSTRACT}

Mechanical properties of a new low-cost unilateral multiaxial external fixator (ATLAS) developed for our group are presented. This is a double-bar fixator assembled with six-degrees-of-freedom connectors so that the entire fixator allows six degrees of freedom between bone fragments. A computational and experimental procedure was developed to obtain the interfragmentary flexibility matrix of the fixator. This study was also undertaken with other three fixators, two imported and one national frequently used in our region. The stiffness of the new fixator was in the range of stiffnesses obtained for the others. Even with the double-bar configuration, which gives good stability and stiffness to the assembly, the ATLAS fixator allows six degrees of freedom between bone fragments, so that the surgeon can reduce the fracture accurately. From the biomechanical point of view, this fixator is ready for clinical evaluation.

Key Words: External fixators, bone fracture, interfragmentary movements.

\section{INTRODUCCIÓN}

Uno de los objetivos de nuestro grupo es el de diseñar, construir y evaluar fijadores externos multiaxiales de bajo costo y buena calidad. En un trabajo previo (Leyton et. al., 2002) se presentaron las características mecánicas de un fijador de una sola barra, que tuvo la rigidez adecuada para tratar fracturas de las extremidades inferiores, pero presentó algunos problemas de estabilidad. Con el objeto de solucionarlos y de poder activar los seis grados de libertad entre los fragmentos óseos, se desarrolló el fijador ATLAS que debe ser utilizado en una configuración de doble barra. Los conectores que componen este fijador permiten seis grados de libertad entre las barras y los clavos que conectan, con lo cual se garantiza que entre los fragmentos de hueso también se puedan activar los seis grados de libertad para que el médico ortopedista pueda reducir adecuadamente la fractura.

La caracterización mecánica se hizo con base en un procedimiento computacional y experimental para determinar la denominada matriz de flexibilidad interfragmentaria de cualquier fijador externo (Leyton et al, 2002). Con dicha matriz es posible predecir todos los movimientos interfragmentarios tridimensionales, los cuales afectan la curación de la fractura (Gardner et al. 1997; Gardner y Weemaes, 1999). Este procedimiento simple permite realizar análisis paramétricos con el modelo computacional calibrado, a diferencia de otros propuestos en la literatura que utilizan solamente pruebas experimentales (Duda et al, 1998). Los resultados del estudio se compararon con los obtenidos para otros tres fijadores: uno nacional (Sardi) ampliamente utilizado en el suroccidente de Colombia y dos importados (Monotubo ${ }^{\circledR}{ }^{1}$ y Orthofix ${ }^{\circledR}$ ProCallus ${ }^{2}$ ). Hasta donde los autores conocen, el conector utilizado en este fijador es el primero que permite activar los seis grados de libertad entre los elementos que conecta.

\footnotetext{
1 Monotube ${ }^{\circledR}$ TRIAX ${ }^{\mathrm{TM}}$ External Fixation System, Dynamic Tube Assembly Blue Ref. 5150-0-070. Striker, Howmedica. Ginebra, Suiza.

2 D.A.F, Orthofix® ProCallus, - Ref. 10028 (Short). Verona, Italia.
} 


\section{MATERIALES Y MÉTODOS}

Se probaron las configuraciones que se presentan en la Figura 1. Los fijadores Monotubo ${ }$, Orthofix ${ }^{\circledR}$ ProCallus y Sardi ya han sido descritos en la literatura técnica. El fijador ATLAS está compuesto, en este caso, por ocho conectores de seis grados de libertad como el que se presenta en la Figura 2.
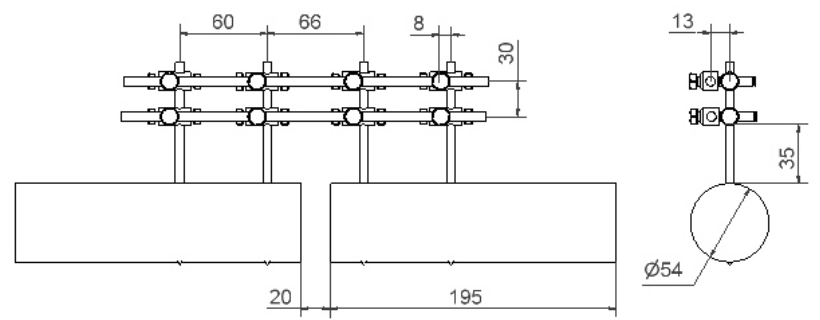

(a) ATLAS
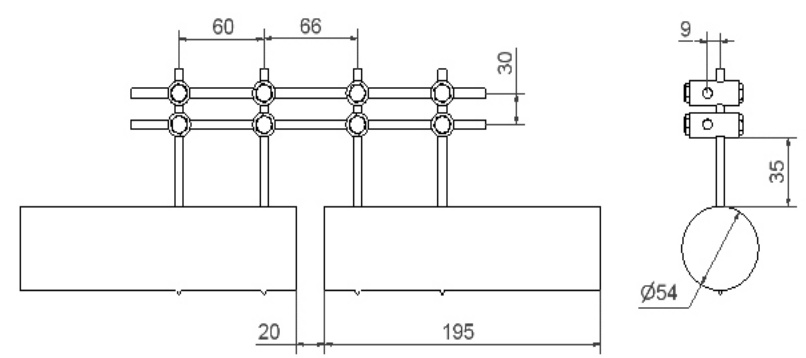

(b) Sardi
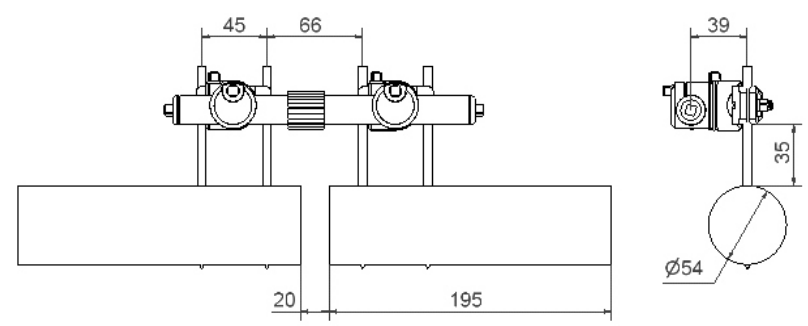

(c) Monotubo
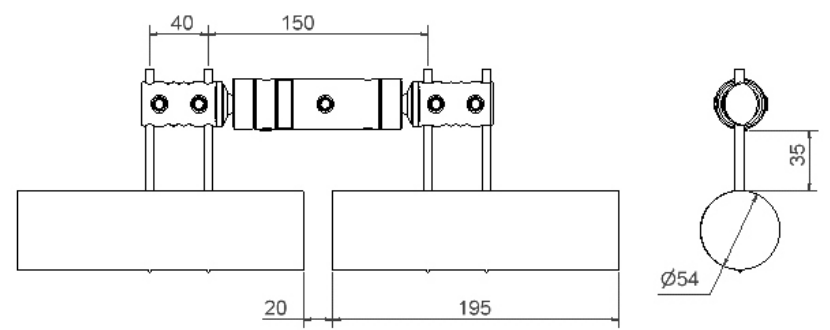

(c) Orthofix $\mathbb{R}$

Unidades en $\mathrm{mm}$.

Mota: Diámetro de clavos $=6 \mathrm{~mm}$

Figura 1. Configuración de los ensambles estudiados
El conector permite desplazamientos ilimitados de las barras y los clavos en las direcciones correspondientes a los grados de libertad 1, 2, 3, 4 y 6 (Figura 2), mientras que en la dirección 5 el desplazamiento está limitado a $10 \mathrm{~mm}$. El rango de los grados de libertad del conjunto depende de factores como el número de clavos y la distancia entre ellos.

La caracterización mecánica de los fijadores se hizo con un procedimiento muy similar al presentado por Leyton et al (2002) tal como se explica a continuación. Se estudió un ensamble de cada fijador montado mediante cuatro clavos en dos barras de nylon ( módulo de elasticidad $E$ $=2757,9 \mathrm{~N} / \mathrm{mm}^{2}$ y relación de Poisson $\mathrm{n}=0.2$ ) que representan los fragmentos de los huesos (Figura 1). El fragmento fijo durante las pruebas se denominó fragmento base y el otro, fragmento móvil.

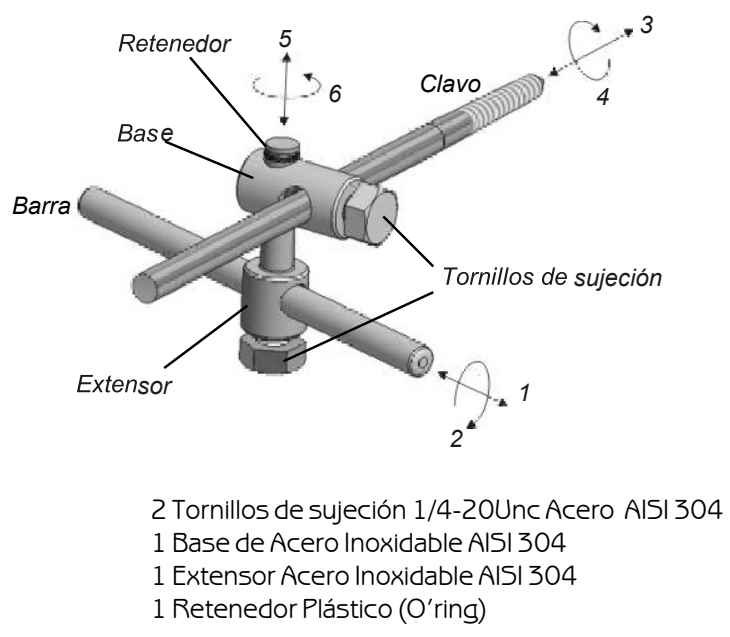

Figura 2. Conector del fijador ATLAS

Experimentalmente cada ensamble fijador-hueso fue sometido a una prueba con carga axial y cuatro pruebas con carga transversal. La prueba con carga axial se realizó en el marco de carga ilustrado en la Figura 3, donde la carga $F$ se incrementó sucesivamente cada 49.1 M (5 kg) hasta 343.3 N (35 kg) y se midieron mediante tres indicadores de carátula Teclock $^{3}$ con precisión de $0.01 \mathrm{~mm}$, los desplazamientos interfragmentarios axiales en puntos ubicados a 
$120^{\circ}$ y a una distancia de $56 \mathrm{~mm}$ del eje axial de los fragmentos. El desplazamiento interfragmentario axial se calculó como el promedio de estas tres mediciones. Además, para garantizar la transmisión de fuerza sin momento, se colocaron balines en los extremos de las barras de nylon. Después del desarrollo de la prueba hasta 343.3 My con el fin de determinar la estabilidad del conjunto, se repitió la prueba hasta $686.7 \mathrm{~N}(70 \mathrm{Kg})$ con incrementos de carga de $98.1 \mathrm{M}(10 \mathrm{Kg})$.

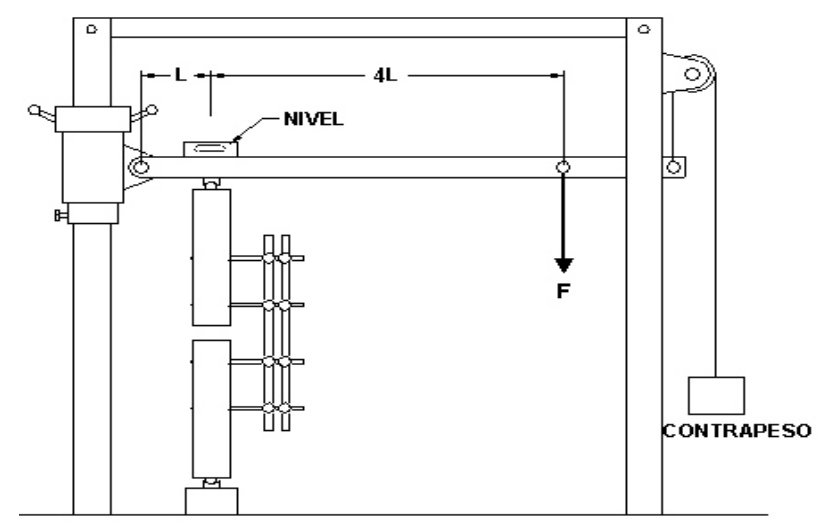

Fiugra 3. Marco de carga para la prueba axial

En las cuatro pruebas con carga trasversal se fijó uno de los fragmentos sobre un soporte de acero (Figura 4) y se colocaron sucesivamente pesos de $9.8 \mathrm{M}(1 \mathrm{~kg})$ hasta completar $58.9 \mathrm{M}(6 \mathrm{~kg})$ en el fragmento móvil en dos posiciones: en la sección del clavo distal (identificada como $\boldsymbol{D}$ ) y en el extremo distal (identificada como $\boldsymbol{E}$ ). Dos de las pruebas se realizaron con la dirección de la carga contenida en el plano del fijador (dirección ML) y las otras dos con el plano del fijador perpendicular al eje de carga (dirección AP). En las pruebas en la dirección AP (identificadas como APD y APE, para diferenciar las posiciones de carga $\boldsymbol{D}$ Y $\boldsymbol{E}$, respectivamente) se midió el desplazamiento en la dirección de la carga en una sección ubicada a

3 TECLOCK, Head quarter / Plant / Magano 2-10-3 Maruta-cho Okaya-shi Magano Pref. 394 - 0042 Japan.
$5 \mathrm{~mm}$ del borde proximal del fragmento móvil (Figura 4). Con el fin de aumentar la sensibilidad de las mediciones, en las pruebas en la dirección ML (identificadas como MLD y MLE), se midió el desplazamiento en la dirección de la carga en el extremo distal del fragmento móvil (Figura 4). Para medir los desplazamientos se utilizó un indicador de carátula Teclock con una precisión de $0.01 \mathrm{~mm}$ y un rango de $0-10 \mathrm{~mm}$.

Cada prueba se realizó cuatro veces consecutivas y para cada una se dibujaron los puntos fuerza vs desplazamiento, a los cuales se ajustaron líneas rectas. La pendiente de la última recta se reporta en resultados como la rigidez experimental, tal como lo recomienda la norma ASTM et al., 2001.

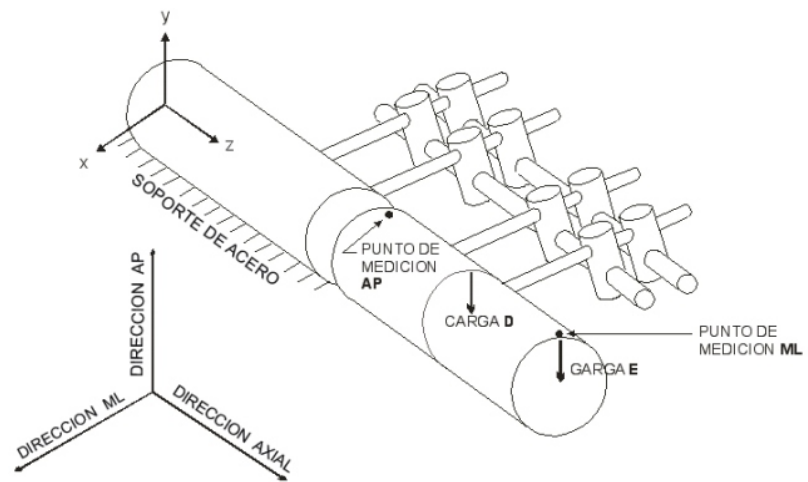

rigura 4. Jecciones de carga y puntos de medicion para una prueba AP

Computacionalmente se analizó un modelo de elementos finitos con el programa comercial Algor ${ }^{\circledR}$. Los fragmentos de nylon se represen̂taron mediante elementos brick de 8 nodos y los demás elementos, es decir los clavos de Schanz, las barras y los conectores, se representaron como elementos viga (Figura 5).

El modelo computacional fue calibrado mediante el ajuste iterativo de las propiedades de los elementos viga, que representan las prensas conectoras, hasta que todos los resultados computacionales fueron cercanos a los experimentales.

4 ALGOR, Inc. 150 Beta Drive. Pittsburgh, PA 15238-2932 USA 


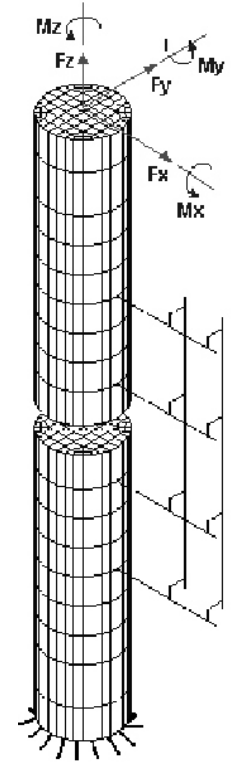

Figura 5. Modelo Computacional

Una vez calibrado, cada modelo computacional se utilizó para determinar la matriz de flexibilidad interfragmentaria, mediante el análisis de una configuración empotrada en el extremo distal del fragmento fijo. En el extremo proximal del fragmento móvil (Figura 4) se aplicaron seis casos de carga, consistentes en tres fuerzas unitarias ( $F x$, Fy y $F z$, en las direcciones $x, y$ y $z$, respectivamente) y tres momentos unitarios ( $M x$, My, $M z$, en las direcciones $x, y$ y $z$, respectivamente). Cada columna de la matriz de flexibilidad estuvo compuesta por los seis desplazamientos interfragmentarios obtenidos para cada caso de carga (traslaciones $d x, d y$ y $d z$ y rotaciones $r x$, ry y $r z$ en las direcciones $x, y$ y $z$ respectivamente). La matriz de rigidez se calculó como la inversa de la matriz de flexibilidad.

La rigidez para cada caso de carga (reportada como rigidez principal) fue calculada como el inverso de cada uno de los elementos de la diagonal principal de la matriz de flexibilidad. Cada valor de rigidez principal tiene el significado físico de fuerza (o momento) necesaria para producir un desplazamiento (o giro) interfragmentario unitario en la respectiva dirección de la aplicación de la fuerza (o momento).
El modelo computacional calibrado se utilizó también para estudiar el efecto de utilizar seis clavos (tres en cada fragmento), mientras los demás parámetros se mantuvieron constantes (Figura 6).

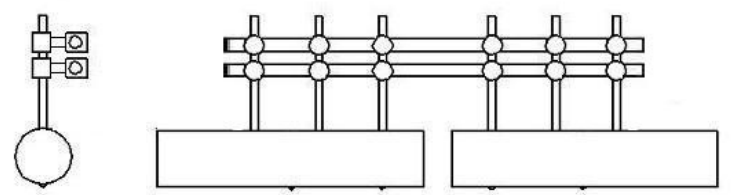

Figura 6. Configuración del fijador con seis clavos

\section{RESULTADOS}

Los conectores del fijador ATLAS mantuvieron su poder de sujeción en el rango de cargas evaluado y se observó un comportamiento consistente y lineal $\left(R^{2}=0.9953\right)$. Además, el fijador mantuvo su estabilidad cuando se sometió a una carga equivalente al peso de una persona de 686.4M $(70 \mathrm{~kg})$. Cuando se repitieron las pruebas para cada configuración y condición de carga se observó un desplazamiento residual (Figura 7). Este comportamiento también se observó en los otros tres fijadores probados y en todos los casos de carga.

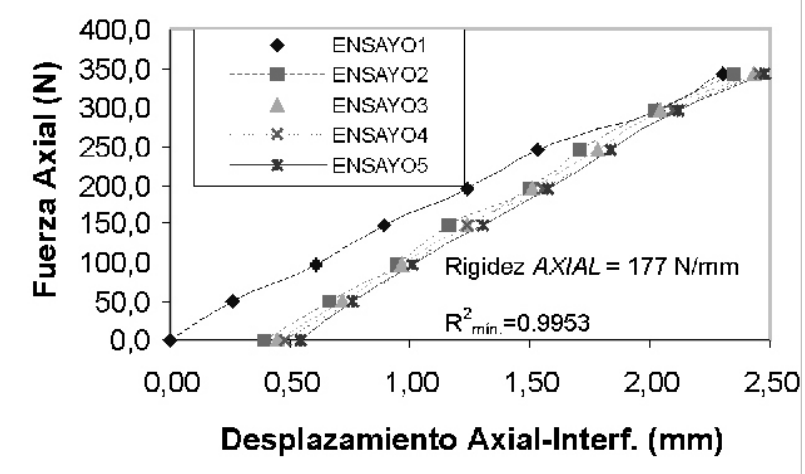

Figura 7. Curvas de carga para la prueba axial Del fijador ATLAS

La rigidez del fijador ATLAS obtenida tanto experimentalmente como con el modelo computacional calibrado (Figuras 8 y 9), fue similar a la rigidez obtenida para los otros fijadores estudiados. 


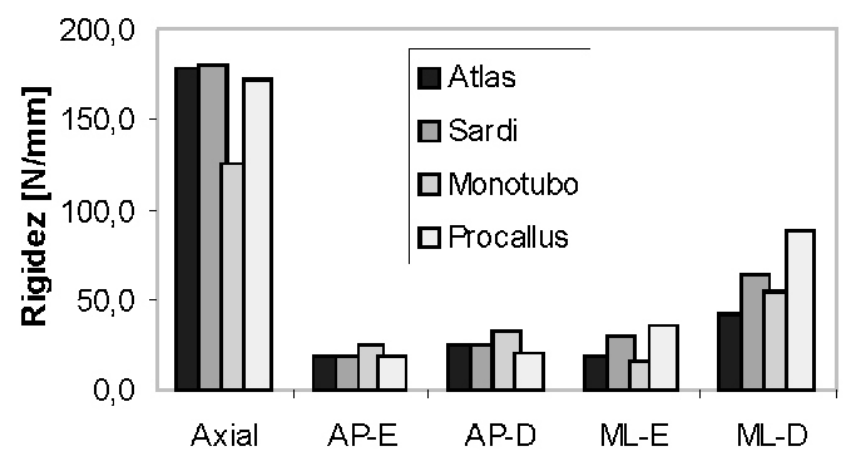

Tipo de Prueba

Figura 8. Comparación entre las rigideces Experimentales para los cuatro fijadores
Después del procedimiento de calibración, la diferencia máxima entre los desplazamientos teóricos y experimentales fue de 11,3\% para el fijador ATLAS, obtenida para el caso de carga APE. Para los otros casos de carga, como el axial, el modelo pudo reproducir la prueba con una diferencia máxima de 3,3\%.

En las matrices de flexibilidad y rigidez (Ecuación 1) se observa un acoplamiento entre los diferentes grados de libertad. Por ejemplo para el fijador ATLAS se produce un giro en el plano del fijador de 0.0037 grados por cada Mewton de carga axial (elemento 5,3 de la matriz de flexibilidad). $\left[\begin{array}{r}d x \\ d y \\ d z \\ r x \\ r y \\ r z\end{array}\right]=\left[\begin{array}{c|c|c|c|c|c}0,0050 & -0,0022 & 0,0005 & 0,0000 & 0,0000 & 0.0000 \\ -0,0022 & 0,0419 & 0,0000 & 0,0000 & 0,0000 & -0.0004 \\ 0,0131 & 0,0075 & 0,0058 & 0,0000 & 0,0001 & 0.0000 \\ 0,0014 & -0,0627 & -0,0022 & 0,0003 & 0,0000 & 0.0000 \\ 0,0133 & -0,0013 & 0,0037 & 0,0000 & 0,0001 & 0.0000 \\ 0,0010 & -0,0239 & 0,0001 & 0,0000 & 0,0000 & 0.0005\end{array}\right]\left[\begin{array}{c}F x \\ F y \\ F z \\ M x \\ M y \\ M z\end{array}\right]$

(a) matriz de flexibilidad
$\left[\begin{array}{r}F x \\ F y \\ F z \\ M x \\ M y \\ M z\end{array}\right]=\left[\begin{array}{r|r|r|r|r|r}433 & 35 & 47 & 10 & -130 & 18 \\ 36 & 91 & 11 & 14 & -17 & 82 \\ 20 & 9 & 581 & 85 & -587 & 6 \\ 7313 & 18457 & 7122 & 6785 & -8663 & 16611 \\ -89650 & -7625 & -43245 & -7197 & 75877 & -3970 \\ 1080 & 4753 & 423 & 724 & -600 & 6506\end{array}\right]\left[\begin{array}{c}d x \\ d y \\ d z \\ r x \\ r y \\ r z\end{array}\right]$

(b) matriz de rigidez

Ecuación 1. Matrices de flexibilidad y de rigidez interfragmentaria para el fijador ATLAS.

(a) Matriz de flexibilidad (b) Matriz de rigidez
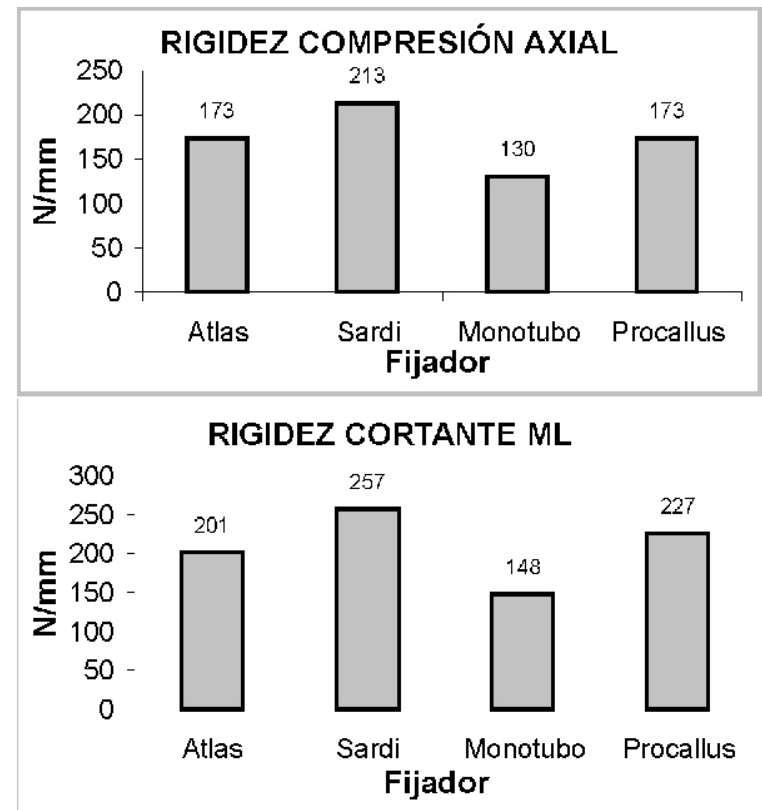
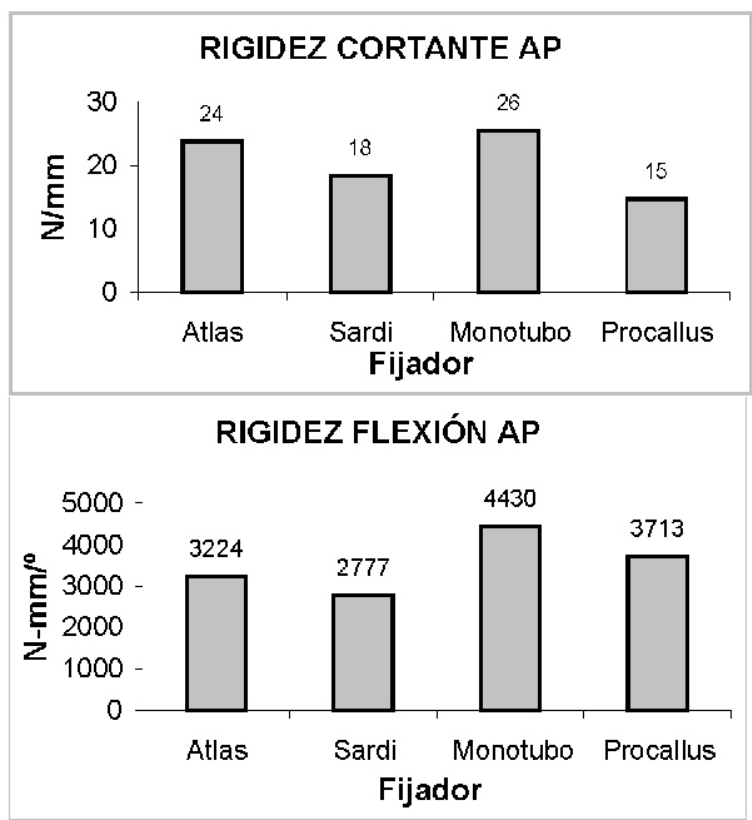

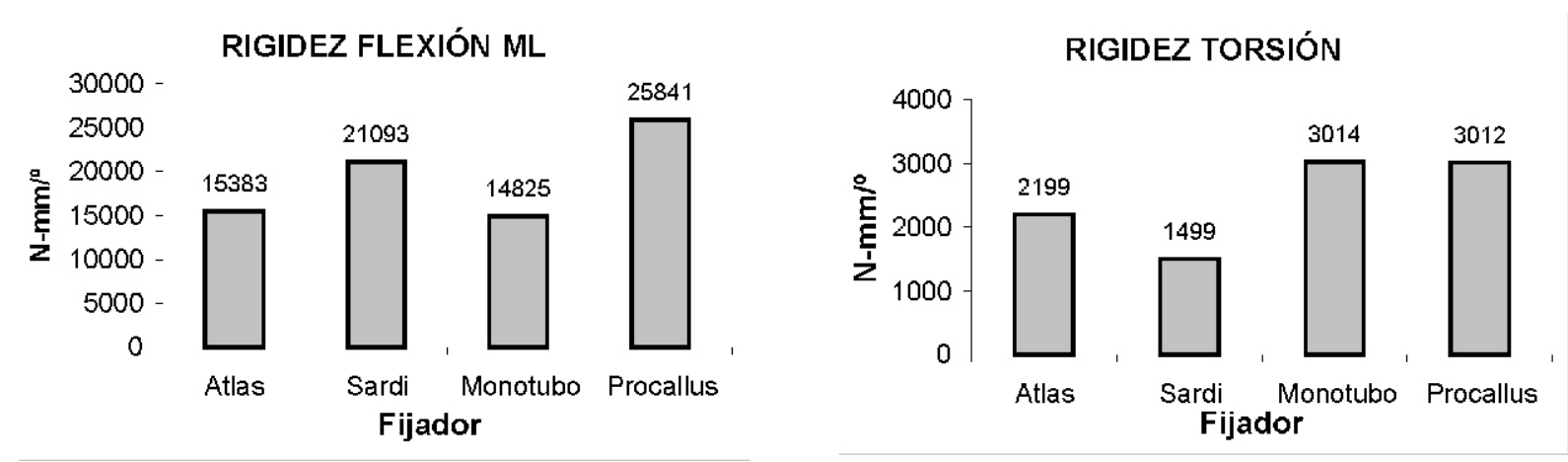

Figura 9. Comparación de las rigideces principales de los cuatro fijadores

Para la configuración del fijador ATLAS con seis clavos se calculó una rigidez axial de $225 \mathrm{M} / \mathrm{mm}$ con el modelo computacional calibrado, lo cual representa un incremento de $33.5 \%$ con respecto a la rigidez con cuatro clavos (Figura 10). Con seis clavos las rigideces en las direcciones APE y MLE aumentaron $18 \%$ y $21 \%$, respectivamente.

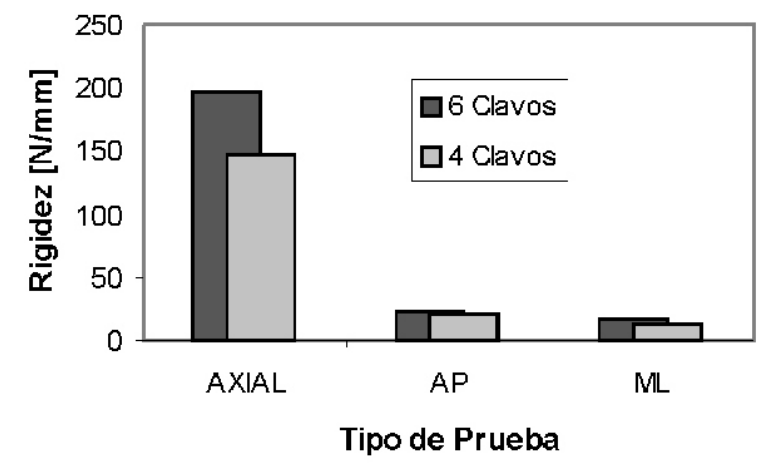

Figura 10. Comparación de la rigidez con configuraciones de cuatro y seis clavos en el fijador ATLAS

\section{DISCUSIÓN Y CONCLUSIONES}

Para todos los casos de carga la rigidez del fijador ATLAS estuvo dentro del rango obtenido para los otros fijadores estudiados, los cuales ya han sido probados clínicamente con buenos resultados. Además, dada su configuración de doble barra, el fijador ATLAS conservó su estabilidad en todas las pruebas, en particular cuando fue sometido a una carga axial máxima de $686.70 \mathrm{M}(70 \mathrm{~kg})$, que sería la esperada en una paciente con una fractura inestable y que apoya el pie con cuidado. Con base en estos resultados se espera que el fijador ATLAS tenga también un comportamiento adecuado en las pruebas clínicas.

Los resultados de este estudio son comparables, ya que las pruebas fueron realizadas en los mismos dispositivos y se trató, hasta donde fue posible, que la configuración fuese similar en todos los montajes. Para el caso del fijador Orthofix ProCallus se obtuvo una rigidez axial de $173 \mathrm{~N} / \mathrm{mm}$, la cual es significativamente similar a $159,5 \mathrm{~N} / \mathrm{mm}$, calculada con base en los desplazamientos interfragmentarios reportados por Gardner et al. (1999) para este mismo fijador. Otros valores de rigidez reportados en la literatura varían ostensiblemente dependiendo de la geometría escogida y el procedimiento utilizado (Yang et al, 2003; Krischak et al, 2002). Éste fue el motivo por el cual se probaron los cuatro fijadores en este estudio.

En todas las pruebas realizadas con estos fijadores se observó un desplazamiento residual después de la primera carga. Éste desplazamiento es típico de los fijadores y por esto la norma ASTM et al 2001 recomienda realizar las pruebas varias veces consecutivas hasta no observar variaciones importantes entre la última y la inmediatamente anterior.

Durante el proceso de calibración del modelo teórico, con base en los resultados 
experimentales, se obtuvieron diferencias máximas en la prueba APE de $11,3 \%$ (en que se reporta la más baja rigidez). Esto se podría atribuir a que el modelo computacional es aproximado, ya que los conectores se representaron como elementos vigas lineales. Una descripción más exacta de estos conectores sólo podría hacerse con elementos de contacto, lo cual es muy costoso computacionalmente ya que un análisis de contacto podría demorar varias horas y aún días. Si se utilizara dicho modelo de contacto se perdería uno de los objetivos del procedimiento, el de ser sencillo. Además, con el modelo actual la diferencia máxima con respecto a las mediciones no sobrepasa el 3,3\% para el caso de carga axial, que se podría considerar como el más representativo para el comportamiento del fijador.

Una revisión de las matrices de flexibilidad y rigidez de los fijadores, indica que existe un acoplamiento importante entre los diferentes grados de libertad. Por ejemplo, cuando el fijador se somete a carga axial, se presenta en el espacio interfragmentario, además del desplazamiento axial, un giro de una sección respecto a otra, lo cual es típico en un fijador unilateral. Recientemente Yang et. al. (2003) caracteriza este acoplamiento mediante el cálculo de la denominada flexibilidad de angulación ("angulation compliance"), la cual define cómo la relación entre el ángulo de giro y el desplazamiento axial interfragmentarios en la prueba axial. Los resultados reportados por Yang et. al. (2003) para fijadores unilaterales están en el rango $0.590 .67 \mathrm{grados} / \mathrm{mm}$. Este parámetro se calculó fácilmente con las matrices de flexibilidad interfragmentaria presentadas en este estudio, ya que es igual a la relación entre la componente de la matriz localizada en la posición $(5,3)$ y aquella de la posición $(3,3)$. Los resultados arrojaron valores entre 0.57 y 0.66 grados $/ \mathrm{mm}$. En un estudio previo (Galvis et al, 2001) se observó que este acoplamiento es menor en los fijadores llizarov debido a su simetría.

Una ventaja significativa del fijador ATLAS es que, aún con una configuración de doble barra que brinda buena estabilidad y rigidez al conjunto, permite tener todos los grados de libertad entre los fragmentos que conecta y entre los clavos y barras. Esto le brinda al médico ortopedista la posibilidad de reducir perfectamente la fractura sin que los clavos deban quedar paralelos o en el mismo plano. Adicionalmente, el diseño mecánico del conector es muy simple y su construcción requiere solamente de operaciones de cilindrado y taladrado, lo que hace que su costo sea bajo. Hasta donde hemos revisado la literatura y la información comercial sobre fijadores no hemos encontrado antecedentes de conectores de seis grados de libertad como el del fijador ATLAS. Desde el punto de vista mecánico se puede afirmar que este fijador está listo para emprender las pruebas clínicas.

\section{Agradecimientos}

El grupo de Biomecánica de la Universidad del Valle agradece el apoyo brindado por Colciencias.

\section{BIBLIOGRAFÍA}

1. ASTM Committee FO4 on Medical an Surgical Materials. Designation $F$ 1541-01. specification and Test Methods for External Esqueletal Fixation Devices, 2001.

2. Duda G., Kirchner H., Wilke H. y Claes L., A method to determine the 3-D stiffness of fracture fixation devices and its application to predict inter-fragmentary movement, Journal of Biomechanics, Vol. 31, pp. 247-252, 1998.

3. Galvis E., Lasso P., Machado A. y García J.J., Computational determination of the $3-D$ stiffness matrix of an llizarov fixator, BED-Vol. 51, Advances in Bioengineering, ASME, 2001.

4. Gardner T.M., Evans M., Hardy J. y Kenwright J., Dynamic inter fragmentary motion in fractures during routine patient activity. Clinical Orthopaedics and Related Resarch, Vol. 336, pp. 216-225, 1997. 
5. Gardner T.M. y Weemaes M., A mathematical stiffness matrix for characterising mechanical performance of the Orthofix DAF, Medical Engineering \& Physics, Vol. 21, Pp. 65-71, 1999.

6. Leyton A., Galvis E., García J.J., Machado A., Echeverry A.A., Modelos de Elementos Finitos para el Análisis y Diseño de Fijadores Externos para Fracturas de Huesos, VI Congreso Colombiano de Elementos Finitos y Modelamiento Mumérico, Universidad Macional, Bogotá, Colombia, 2002
7. Krischak G.D., Janousek, Wolf S., Angat P., Kinsi L. y Claes L., Effects of one - plane and two plane external fixation on sheep osteotomy healing and complications, Clinical Biomechanics, Vol. 17, p.p. 470 476, 2002.

8. Yang L., Magayam 5., Saleh M., Stiffness characteristics and interfragmentary displacements with different hybrid external fixators. Clinical Biomechanics, Vol 18, PP. 166-172, 2003.

Anexo A. Matrices de flexibilidad y de rigidez interfragmentarias de los otros fijadores estudiados.

\section{A1. Monotubo}

(a) Matriz de flexibilidad

$\left[\begin{array}{r}d x \\ d y \\ d z \\ r x \\ r y \\ r z\end{array}\right]=\left[\begin{array}{c|c|c|c|c|c}0,0068 & 0,0005 & 0,0009 & 0,0000 & 0,0000 & 0,0000 \\ 0,0005 & 0,0391 & -0,0001 & 0,0000 & 0,0000 & -0,0003 \\ 0,0179 & -0,0023 & 0,0077 & 0,0000 & 0,0001 & 0,0000 \\ 0,0000 & -0,0463 & 0,0006 & 0,0002 & 0,0000 & 0,0000 \\ 0,0138 & 0,0000 & 0,0051 & 0,0000 & 0,0001 & 0,0000 \\ -0,0004 & -0,0176 & 0,0000 & 0,0000 & 0,0000 & 0,0003\end{array}\right]\left[\begin{array}{c}F x \\ F y \\ F z \\ M x \\ M y \\ M z\end{array}\right]$

\section{A2. Procallus}

$\left.\begin{array}{r}d x \\ d y \\ d z \\ r x \\ r y \\ r z\end{array}\right]=\left[\begin{array}{l|l|l|l|l|l}0,0044 & 0,0000 & 0,0005 & 0,0000 & 0,0000 & 0,0000 \\ 0,0000 & 0,0676 & 0,0000 & 0,0000 & 0,0000 & -0,0003 \\ 0,0109 & 0,0000 & 0,0058 & 0,0000 & 0,0001 & 0,0000 \\ 0,0000 & -0,0552 & 0,0000 & 0,0003 & 0,0000 & 0,0000 \\ 0,0079 & 0,0000 & 0,0030 & 0,0000 & 0,0000 & 0,0000 \\ 0,0000 & 0,0181 & 0,0000 & 0,0000 & 0,0000 & 0,0003\end{array}\right]\left[\begin{array}{c}F x \\ F y \\ F z \\ M x \\ M y \\ M z\end{array}\right]$

(a) Matriz de flexibilidad

$\left.\begin{array}{l}F x \\ F y \\ F z \\ M x \\ M y \\ M z\end{array}\right]$

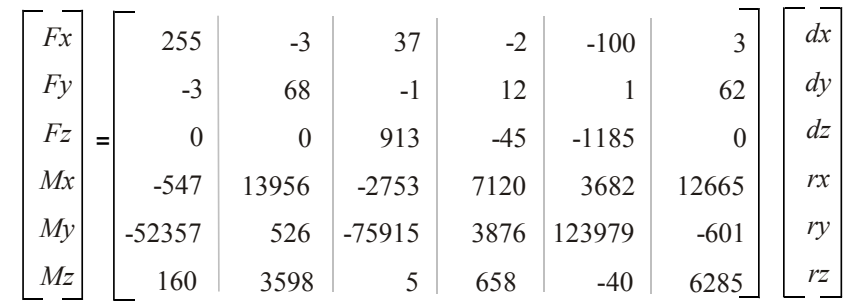

(b) Matriz de rigidez

\section{A3. Sardi}

$\left[\begin{array}{r}d x \\ d y \\ d z \\ r x \\ r y \\ r z\end{array}\right]=\left[\begin{array}{c|c|c|c|c|c}0,0039 & -0,0012 & 0,0005 & 0,0000 & 0,0000 & 0,0000 \\ -0,0012 & 0,0544 & 0,0003 & -0,0001 & 0,0000 & -0,0006 \\ 0,0092 & 0,0058 & 0,0047 & 0,0000 & 0,0000 & 0,0000 \\ 0,0009 & -0,0720 & -0,0017 & 0,0004 & 0,0000 & 0,0000 \\ 0,0095 & -0,0008 & 0,0027 & 0,0000 & 0,0000 & 0,0000 \\ 0,0005 & 0,0325 & 0,0000 & 0,0000 & 0,0000 & 0,0007\end{array}\right]\left[\begin{array}{c}F x \\ F y \\ F z \\ M x \\ M y \\ M z\end{array}\right]$

(a) Matriz de flexibilidad

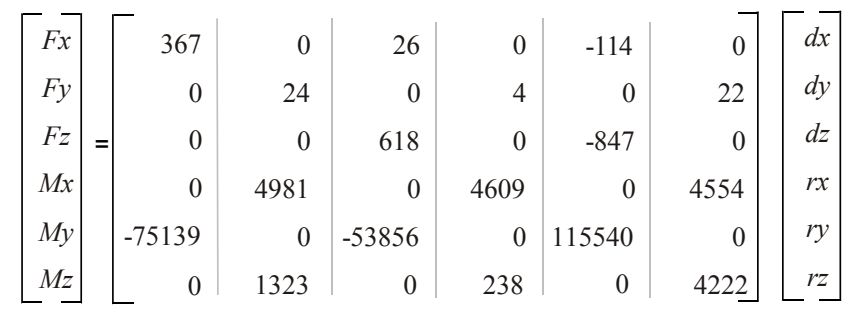

(b) Matriz de rigidez

\section{A3. Sardi}

$\left[\begin{array}{r}F x \\ F y \\ F z \\ M x \\ M y \\ M z\end{array}\right]=\left[\begin{array}{r|r|r|r|r|r}545 & 24 & 32 & 7 & -147 & 14 \\ 25 & 72 & 2 & 13 & 7 & 60 \\ 0 & 0 & 518 & 48 & -507 & 0 \\ 5013 & 14395 & 3141 & 5654 & 4415 & 12057 \\ -109061 & 4811 & 35779 & 4389 & 79421 & 2752 \\ 835 & 3490 & 67 & 633 & -247 & 4426\end{array}\right]\left[\begin{array}{r}d x \\ d y \\ d z \\ r x \\ r y \\ r z\end{array}\right]$

(b) Matriz de rigidez 